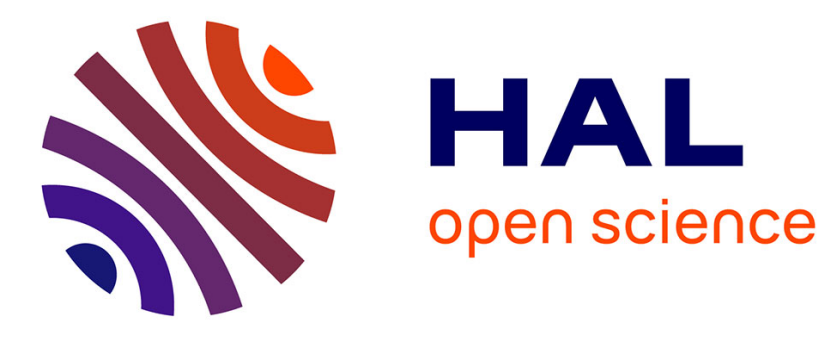

\title{
An Algorithmic Construction of Four-Level Response Surface Designs
}

Christos Koukouvinos, Kalliopi Mylona, Dimitris E Simos, Anna Skountzou

\section{To cite this version:}

Christos Koukouvinos, Kalliopi Mylona, Dimitris E Simos, Anna Skountzou. An Algorithmic Construction of Four-Level Response Surface Designs. Communications in Statistics - Simulation and Computation, 2009, 38 (10), pp.2152-2160. 10.1080/03610910903259634 . hal-00524863

\section{HAL Id: hal-00524863 https://hal.science/hal-00524863}

Submitted on 9 Oct 2010

HAL is a multi-disciplinary open access archive for the deposit and dissemination of scientific research documents, whether they are published or not. The documents may come from teaching and research institutions in France or abroad, or from public or private research centers.
L'archive ouverte pluridisciplinaire HAL, est destinée au dépôt et à la diffusion de documents scientifiques de niveau recherche, publiés ou non, émanant des établissements d'enseignement et de recherche français ou étrangers, des laboratoires publics ou privés. 


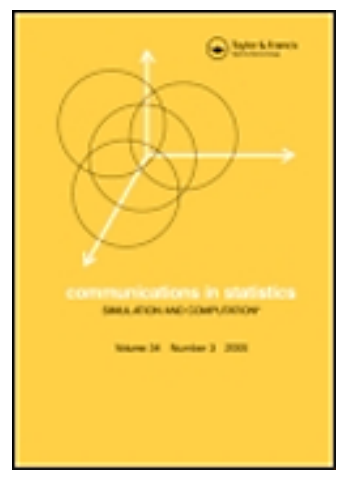

\section{An Algorithmic Construction of Four-Level Response Surface Designs}

\begin{tabular}{|c|c|}
\hline Journal: & Communications in Statistics - Simulation and Computation \\
\hline Manuscript ID: & LSSP-2008-0313.R1 \\
\hline Manuscript Type: & Original Paper \\
\hline $\begin{array}{r}\text { Date Submitted by the } \\
\text { Author: }\end{array}$ & 28-Jul-2009 \\
\hline Complete List of Authors: & $\begin{array}{l}\text { Koukouvinos, Christos; National Technical University of Athens } \\
\text { Mylona, Kalliopi; National Technical University of Athens } \\
\text { Simos, Dimitris; National Technical University of Athens } \\
\text { Skountzou, Anna; National Technical University of Athens }\end{array}$ \\
\hline Keywords: & $\begin{array}{l}\text { Response Surface Designs, Rotatability, Genetic Algorithms, } \\
\text { Efficiency, Optimization }\end{array}$ \\
\hline Abstract: & $\begin{array}{l}\text { Response surface methodology is widely used for developing, } \\
\text { improving and optimizing processes in various fields. In this } \\
\text { paper we present a method for constructing four-level design } \\
\text { matrices in order to explore and optimize response surfaces where } \\
\text { the predictor variables are each at four equally spaced levels, by } \\
\text { utilizing a genetic algorithm. The produced designs achieve both } \\
\text { properties of near-rotatability and estimation efficiency. }\end{array}$ \\
\hline \multicolumn{2}{|c|}{$\begin{array}{l}\text { Note: The following files were submitted by the author for peer review, but cannot be converted } \\
\text { to PDF. You must view these files (e.g. movies) online. }\end{array}$} \\
\hline koukouvinos.tex & \\
\hline
\end{tabular}




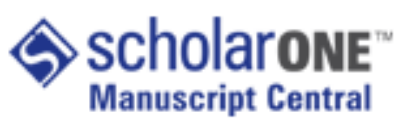




\title{
AN ALGORITHMIC CONSTRUCTION OF FOUR-LEVEL RESPONSE SURFACE DE- SIGNS
}

C. Koukouvinos, K. Mylona, D. E. Simos and A. Skountzou

Department of Mathematics

National Technical University of Athens

Zografou, Athens, Greece 15773

ckoukouv@math.ntua.gr, kmylona@math.ntua.gr,dsimos@math.ntua.gr and askount@central.ntua.gr

Key Words: response surface designs; four-level factors; rotatability; genetic algorithms; efficiency; optimization.

Running Head: CONSTRUCTION OF RESPONSE SURFACE DESIGNS

AMS Subject Classification: 62K20.

\begin{abstract}
Response surface methodology is widely used for developing, improving and optimizing processes in various fields. In this paper we present a method for constructing four-level design matrices in order to explore and optimize response surfaces where the predictor variables are each at four equally spaced levels, by utilizing a genetic algorithm. The produced designs achieve both properties of near-rotatability and estimation efficiency.
\end{abstract}

\section{INTRODUCTION}

Response surface methodology is used in experiments in which the main interests are to determine the relationship between the response and the settings of a group of experimental factors and to find the combination of the factor levels that gives the best expected response. Response surfaces can also provide information about the rate of change of the response variable and indicate the interactions between the treatment factors. This class of designed experiments has a wide range of applications in industrial and chemical engineering, agricultural experiments and biotechnological processes (Box and Draper, 1987, Edmondson, 1991, Gilmour, 2004, Gilmour, 2006, Khuri and Cornell, 1996, Myers and Montgomery, 2002). 
In this paper we mainly focus on the construction of four-level response surface designs. In such designs the design matrix columns are constituted of combinations of four distinct symbols and correspond to the treatment factors, each at four equally spaced quantitative levels. Any combination of the levels of all factors under consideration is called a treatment combination. Let $\mathbf{X}=\left[x_{1}, x_{2}, \ldots, x_{k}\right]$ be the design matrix of the experiment in which, each row represents the $\mathrm{n}$ treatment combinations and each column gives the sequence of factor levels. For each factor, all level values are of equal interest and each experimental result should have equal influence. Thus we consider designs with the equal occurrence property, where all columns consist of $n / 4$ elements equal to $1, n / 4$ elements equal to $-1, n / 4$ elements equal to $1 / 3, n / 4$ elements equal to $-1 / 3$, when $n$ is multiple of four. The designs with the equal occurrence property are called balanced designs. Although four-level factors appear often in experimental problems, a minor work has be done in this area of response surface designs (Edmondson, 1991, Gilmour, 2004, Gupta and Dey, 1975).

The paper is organized as follows. In Section 2. the concepts and the measures of rotatability and efficiency of response surface designs are defined. The introduced method for the construction of four-level response surface designs is presented in Section 3., while the obtained results and an application are illustrated in Section 4.

\section{MODEL AND DESIGN CRITERIA}

Suppose we want to test the effects of $k$ predictor variables, coded to $x_{1}, x_{2}, \ldots, x_{k}$, on a response variable $y$ subject to random error. Generally the first attempt is to approximate the shape of the response surface by fitting a first-order model to the response,

$$
y=\beta_{0}+\sum_{j=1}^{k} \beta_{j} x_{j}+\varepsilon,
$$

where $\beta_{0}, \beta_{j}, j=1, \ldots, k$ are unknown parameters and $\varepsilon$ is a random error term. When the first-order model appears inadequate to describe the true relationship between the response and the predictor variables due to the existence of surface curvature, it is upgraded to a second-order model 


$$
y=\beta_{0}+\sum_{j=1}^{k} \beta_{j} x_{j}+\sum_{j=1}^{k} \beta_{j j} x_{j}^{2}+\underbrace{\sum_{i=1}^{k} \sum_{j=1}^{k}}_{i<j} \beta_{i j} x_{i} x_{j}+\varepsilon,
$$

where $\beta_{0}, \beta_{j}, j=1, \ldots, k, \beta_{i j}, i=1, \ldots, k, j=1, \ldots, k$, are unknown parameters and $\varepsilon$ is a random error term.

Two of the most important characteristics that a response surface design should possess is rotatability and efficiency.

The concept of rotatability was introduced by Box and Hunter (1957). A $k$-dimensional design is called rotatable if the variance of the response estimated by the fitted polynomial at the point $\left(x_{1}, \ldots, x_{k}\right), \operatorname{Var}[\hat{Y}(\mathbf{x})]$, is a function only of $\rho^{2}=\sum_{i=1}^{k} x_{i}^{2}$. Such a design insures that the estimated response has a constant variance at all points that are equidistant from the design center. One of the desirable features of rotatability is that the quality of the prediction, as measured by the magnitude of $\operatorname{Var}[\hat{Y}(\mathbf{x})]$, is invariant to any rotation of the coordinate axes in the space of the input variables. In cases where exact rotatability is unattainable, it is important to measure how rotatable a design is. Khuri (1988), Draper and Guttman (1988), and Draper and Pukelsheim (1990) proposed measures to test the near rotatability of a design. In this framework we use the rotatability measure $Q^{*}$ provided by Draper and Pukelsheim (1990) and given by the equation

$$
Q^{*}=\frac{\left\|\overline{\mathbf{A}}-\mathbf{V}_{\mathbf{0}}\right\|^{2}}{\left\|\mathbf{A}-\mathbf{V}_{\mathbf{0}}\right\|^{2}}=\frac{\operatorname{tr}\left(\overline{\mathbf{A}}-\mathbf{V}_{\mathbf{0}}\right)^{2}}{\operatorname{tr}\left(\mathbf{A}-\mathbf{V}_{\mathbf{0}}\right)^{2}}
$$

where $\overline{\mathbf{A}}$ is the rotatable component of the moment matrix $\mathbf{A}=n^{-1} \mathbf{X}^{\prime} \mathbf{X}$ and $\mathbf{V}_{\mathbf{0}}$ consists of a one in the $(1,1)$ position and zeros elsewhere. It is $Q^{*} \leqslant 1$ and equality stands when the design is rotatable. For more details see Draper and Pukelsheim (1990).

Beyond testing the near rotatability of the designs in order to compare them, it is also needed to have an estimation efficiency measure for the same purpose. Box and Draper (1971) discussed as a measure of design efficiency the choice of a design on the basis of 
maximizing the determinant of the information matrix. In this paper we adopt the following $D$ criterion for determining the overall efficiency for estimating the set of the effects

$$
\left|\mathbf{W}^{\prime} \mathbf{W}\right|^{1 / k}
$$

where $\mathbf{W}=\left[x_{0} /\left\|x_{0}\right\|, x_{1} /\left\|x_{1}\right\|, \ldots, x_{k} /\left\|x_{k}\right\|\right], x_{0}$ stands for the vector with all elements equal to 1 , and $x_{i}$ is the coefficient vector of the $i$-th effect, $i=1, \ldots, k$. Since the columns of $\mathbf{W}$ are standardized, the $D$ criterion achieves its maximum value, which equals to 1 , if and only if the $x_{i}$ are orthogonal to each other. More details can be found in Wang and $\mathrm{Wu}(1995)$.

\section{BUILDING RESPONSE SURFACE DESIGNS BY MEANS OF GENETIC ALGO- RITHMS}

Genetic algorithms form a powerful metaheuristic that mimicks processes from the Theory of Evolution to establish search algorithms by defining algorithmic analogues of biological concepts such as reproduction, crossover and mutation. Genetic Algorithms were introduced by John Holland (1975) aiming to design an artificial system having properties similar to natural systems. In this paper, we assume some basic familiarity with Genetic Algorithm concepts. The concepts necessary for a description of the Genetic Algorithm (GA) can be found in Goldberg (1989), in Stefanie Forrest's article (1993) and in the Handbook of Genetic Algorithms edited by Davis (1991).

GAs are attractive because of their robustness and flexibility in terms of a computer implementation and, mathematically, they do not require a differentiable objective function thereby reducing the chance of reporting local optima. Some earlier attempts utilizing a GA in the construction of response surface designs has been given by Drain et al. (2004). However, this approach, while promising, lacked of an efficient coding of the chromosomes i.e. the number of the experimental runs forming the design. In particular, the authors proposed utilizing and constructing the whole design; thus restricting the GA to evolve in finding optimal response surface designs in several cases. A successful reduction in terms of computational complexity of an efficient representation of the candidate design, has been 
proposed in Koukouvinos et al. (2007), Koukouvinos et al. (2008) and Koukouvinos et al. (2009) in a similar field of computational design theory with strong connection to statistical applications. In these applications, the authors integrated as a core ingredient of the GA the use of sequential juxtaposition of suitable generators, either forming circulant matrices (Koukouvinos et al., 2007) or block circulant matrices (Koukouvinos et. al., 2008, Koukouvinos et al., 2009).

In the present paper, the respective generators considered in the case of response surface designs are the $n / 4$ column vectors which in the process form block circulant matrices of order $q$, when constructing an $n \times q$ response surface design. However, in all previous constructions the generators, more precisely the genes forming a generator, consisted of binary variables since a two-level design was under development. In the case of response surface designs, the genes constitute of four possible values representing the four levels of the designs. A suitable encoding to binary variables was needed since the genetic operators behave better in binary arithmetic (Goldberg, 1989). The answer to this vital question found in the field of Combinatorics and Computer Science in terms of representing a 2-bit Gray Code, $G C=\{00,01,11,10\}$. For more details, on Gray Codes we refer the interested reader to Carla (1997). More precisely, we mapped each level of the design to a codeword of the 2-bit Gray Code, i.e. $\{-1,-1 / 3,1 / 3,1\} \rightarrow\{00,01,11,10\}$, thus transforming the problem on its binary equivalent which allowed us to carry on with the next stages of utilizing a GA.

We defined the basic genetic operation, crossover, that splits a pair of binary integers at a random position and combines the head of one with the tail of the other and vice versa. Additional operations, such as inverting a section of the binary representation (inversion) or randomly changing the state ( 0 or 1$)$ of individual bits (mutation), also transform the population. Before each such cycle (generation), population members are selected on the basis of their fitness (the value of the objective function for that solution) to be the "parents" of the new generation. Last, but not least, the crucial choice of the objective function subject to be optimized arise naturally from the theoretical framework of rotatable designs. Thus, a design was considered optimal or near-optimal when a rotatable, respectively near-rotatable, 
response surface design was found.

\section{RESULTS AND APPLICATIONS}

\subsection{THE RESULTS}

In this section we present the results of the construction method for four-level response surface designs described previously. In Table $1, k$ stands for the number of the experimental factors and $n$ for the number of the performed runs, while in the next two columns the achieved values for the $Q^{*}$ and the $D$ criterion are listed.

\begin{tabular}{|c|c|c|c|c|c|c|c|c|c|c|c|}
\hline$k$ & $n$ & $Q^{*}$ & $D$ & $k$ & $n$ & $Q^{*}$ & $D$ & $k$ & $n$ & $Q^{*}$ & $D$ \\
\hline 2 & 8 & 0.959854 & 0.688198 & 4 & 64 & 0.984979 & 0.752422 & 6 & 80 & 0.964286 & 0.729376 \\
\hline 2 & 12 & 0.987357 & 0.720681 & 4 & 68 & 0.978612 & 0.752464 & 6 & 84 & 0.966084 & 0.731742 \\
\hline 2 & 16 & 0.989625 & 0.730767 & 4 & 72 & 0.987028 & 0.755138 & 6 & 88 & 0.958951 & 0.737689 \\
\hline 2 & 20 & 0.983030 & 0.728812 & 4 & 76 & 0.974130 & 0.757333 & 7 & 40 & 0.904364 & 0.477906 \\
\hline 2 & 24 & 0.989625 & 0.730767 & 4 & 80 & 0.984413 & 0.758021 & 7 & 44 & 0.925751 & 0.547346 \\
\hline 2 & 28 & 0.988432 & 0.729770 & 5 & 24 & 0.921252 & 0.560310 & 7 & 48 & 0.912101 & 0.567746 \\
\hline 2 & 32 & 0.989625 & 0.730767 & 5 & 28 & 0.931478 & 0.624055 & 7 & 52 & 0.912179 & 0.600130 \\
\hline 2 & 36 & 0.987580 & 0.730165 & 5 & 32 & 0.921772 & 0.637922 & 7 & 56 & 0.926475 & 0.618743 \\
\hline 2 & 40 & 0.989625 & 0.730767 & 5 & 36 & 0.941109 & 0.664694 & 7 & 60 & 0.928214 & 0.641472 \\
\hline 2 & 44 & 0.988255 & 0.730364 & 5 & 40 & 0.949874 & 0.687925 & 7 & 64 & 0.944080 & 0.653896 \\
\hline 3 & 12 & 0.957796 & 0.672742 & 5 & 44 & 0.956078 & 0.701092 & 7 & 68 & 0.931776 & 0.662358 \\
\hline 3 & 16 & 0.974468 & 0.723329 & 5 & 48 & 0.961182 & 0.709732 & 7 & 72 & 0.941300 & 0.673442 \\
\hline 3 & 20 & 0.974757 & 0.725922 & 5 & 52 & 0.959283 & 0.722619 & 7 & 76 & 0.949344 & 0.679945 \\
\hline 3 & 24 & 0.978582 & 0.735271 & 5 & 56 & 0.959163 & 0.721522 & 7 & 80 & 0.949483 & 0.692045 \\
\hline 3 & 28 & 0.980806 & 0.741253 & 5 & 60 & 0.964248 & 0.727228 & 7 & 84 & 0.944670 & 0.700672 \\
\hline 3 & 32 & 0.982045 & 0.740266 & 5 & 64 & 0.971776 & 0.738562 & 7 & 88 & 0.933912 & 0.704588 \\
\hline 3 & 36 & 0.984743 & 0.744575 & 5 & 68 & 0.968770 & 0.739369 & 7 & 92 & 0.952219 & 0.713946 \\
\hline 3 & 40 & 0.985867 & 0.745508 & 5 & 72 & 0.974636 & 0.742555 & 8 & 48 & 0.886695 & 0.438586 \\
\hline 3 & 44 & 0.981693 & 0.743881 & 5 & 76 & 0.965921 & 0.745443 & 8 & 52 & 0.892769 & 0.491582 \\
\hline 3 & 48 & 0.989398 & 0.746684 & 5 & 80 & 0.972586 & 0.753905 & 8 & 56 & 0.908462 & 0.518264 \\
\hline 4 & 16 & 0.901968 & 0.565480 & 6 & 32 & 0.909902 & 0.525207 & 8 & 60 & 0.907114 & 0.557043 \\
\hline 4 & 20 & 0.942385 & 0.655520 & 6 & 36 & 0.911722 & 0.563752 & 8 & 64 & 0.918131 & 0.574709 \\
\hline 4 & 24 & 0.936037 & 0.682163 & 6 & 40 & 0.927589 & 0.608824 & 8 & 68 & 0.924789 & 0.611768 \\
\hline 4 & 28 & 0.962690 & 0.700950 & 6 & 44 & 0.941761 & 0.628792 & 8 & 72 & 0.931797 & 0.612020 \\
\hline 4 & 32 & 0.970536 & 0.716913 & 6 & 48 & 0.939694 & 0.646278 & 8 & 76 & 0.925060 & 0.631981 \\
\hline 4 & 36 & 0.972616 & 0.722305 & 6 & 52 & 0.936277 & 0.667183 & 8 & 80 & 0.919916 & 0.643375 \\
\hline 4 & 40 & 0.970273 & 0.734296 & 6 & 56 & 0.938638 & 0.678255 & 8 & 84 & 0.933816 & 0.655232 \\
\hline 4 & 44 & 0.961606 & 0.735100 & 6 & 60 & 0.954363 & 0.690468 & 8 & 88 & 0.934001 & 0.667397 \\
\hline 4 & 48 & 0.984123 & 0.741785 & 6 & 64 & 0.955500 & 0.705109 & 8 & 92 & 0.942649 & 0.671583 \\
\hline 4 & 52 & 0.979102 & 0.750763 & 6 & 68 & 0.950524 & 0.712883 & 8 & 96 & 0.944903 & 0.683993 \\
\hline 4 & 56 & 0.977446 & 0.745832 & 6 & 72 & 0.952703 & 0.715155 & 8 & 100 & 0.945041 & 0.689233 \\
\hline 4 & 60 & 0.967011 & 0.748081 & 6 & 76 & 0.950139 & 0.724041 & & & & \\
\hline
\end{tabular}

Table 1: Results for the constructed four-level response surface designs.

From the above results we note that the $Q^{*}$ values fluctuate between $88.67 \%$ and $98.96 \%$ and the arithmetical mean equals to $95.30 \%$, while the maximum and the minimum values 
of the $D$-criterion are $75.80 \%$ and $43.86 \%$, respectively, with the arithmetical mean equal to 68.18\%. Also, Koshal's designs (Box and Draper, 2007, Koshal, 1933) are occasionally of use in response surface work. A polynomial of degree $d$ in $k$ variables contains $p=(k+d) ! /(d ! k !)$ coefficients. An example of a design that contains exactly this number of runs for the case $d=2$ was given in Koshal (1933). Koshal 's design is extended to any order d and to any number of predictor variables $k$. However, for the third-order Koshal design in 3 fourlevel predictor variables with 20 runs, given in page 504 of Box and Draper (2007), we calculate the corresponding values of $Q^{*}$ and $D$-criterion, which are equal to 0.3150 and 0.2613 , respectively.

In Edmondson (1991) response surface designs with four-level factors were constructed, using classical methods of construction based on fractional replications and pseudo factorial and it was shown that fractionation and confounding can be used to obtain flexible families of designs that are convenient to use and have useful blocking systems. Response surface designs with some four-level factors were also constructed from two-level factorial and fractional factorial designs through pseudo-factors, in Gilmour (2004). The designs appeared in the above papers, although they do not include many factors, have useful properties. In Gupta and Ney (1975) second order rotatable designs with factors each at four levels were constructed via balanced incomplete block designs. Although these designs are rotatable, they demand a large number of runs in order to be constructed.

The approach to construct response surface designs by means of optimization is of current interest. Our efforts were concentrated on the two criteria, $Q^{*}$ and $D$ via a metaheuristic search, because high values of them ensure that the designs are near-rotatable and efficient for estimating the set of the effects The flexibility of genetic algorithms allows different objective functions to be optimized. Therefore, if another criterion was under consideration our genetic algorithm could be applied in a similar manner. According to this, with the appropriate objective function, our algorithm could be used for the construction of orthogonally blocked four-level second order designs and this could be the basis of future work.

As a conclusion, our construction method manages to generate near-rotatable and ef- 
ficient four-level response surface designs for $2 \leq k \leq 8$ factors, with a small number of required runs. The tables with the designs that we achieved to construct, as well as their corresponding values to the above optimality criteria are given in Prof. C. Koukouvinos web site, http://www.math.ntua.gr/へckoukouv/.

\subsection{AN ILLUSTRATIVE EXAMPLE}

Suppose that an experimenter wants to test how three experimental factors, each at four levels, influence the response vector $y$, and has also the goal to optimize the response. In order to fit a second-order model to the data, a response surface design with three factors $A, B, C$ and 20 runs, constructed as described in the previous section, could be used. The corresponding design matrix is

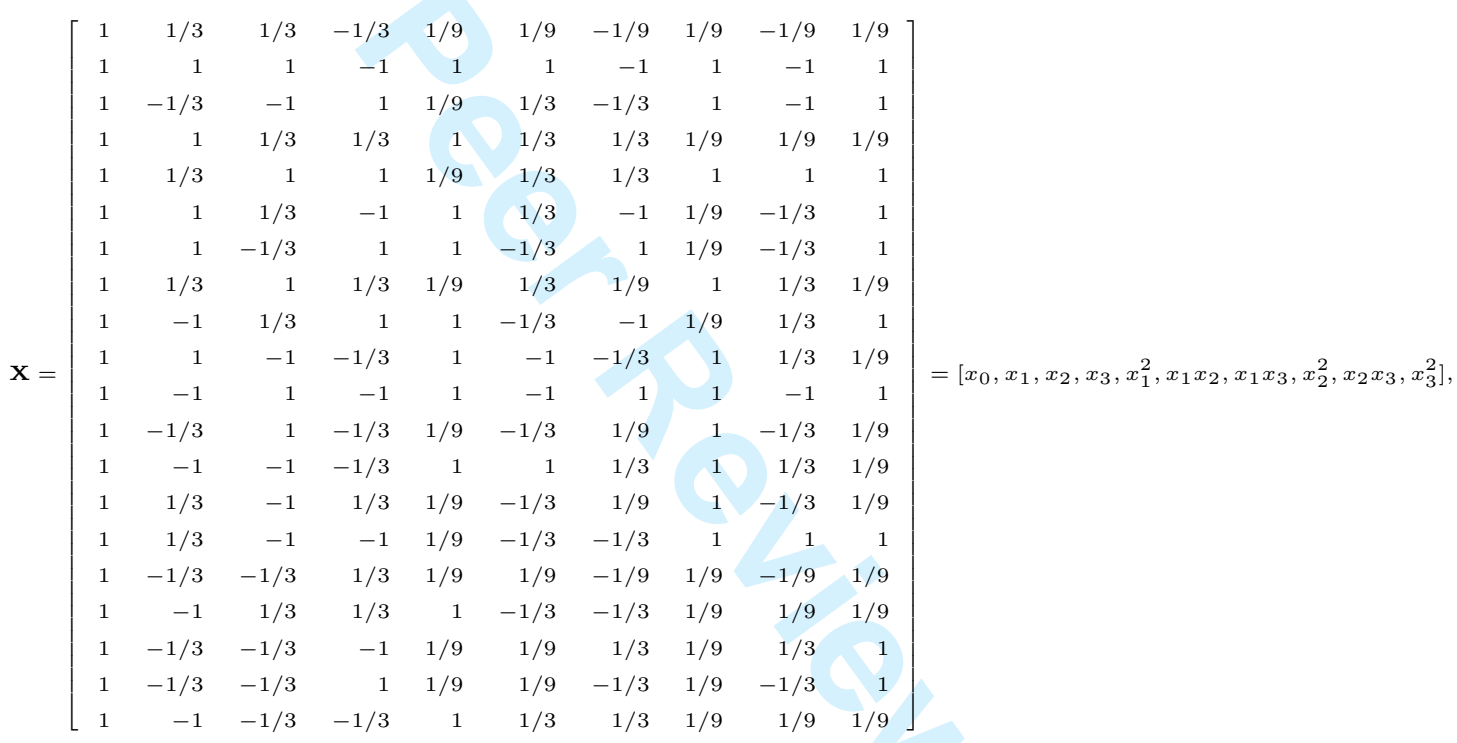

where the first column stands for the general mean.

In order to test our results we use simulated data obtained from the second-order model, in a similar form to model given by Equation 2.,

$$
y=0.5+3 x_{1}+4 x_{2}+3 x_{1}^{2}+3 x_{1} x_{2}+3 x_{2}^{2}+\varepsilon,
$$


where $\varepsilon \sim N_{20}\left(0_{20}, I_{20}\right)$ (i.e., $\varepsilon$ is i.i.d. to a multivariate normal distribution with mean vector zero and with a variance matrix the identity matrix). A response $y$, obtained by using the above simulated model, is

$$
\begin{aligned}
y= & {[2.6451,14.2977,0.8196,8.6477,10.1604,9.4004,4.5216,8.8291,0.2194,2.1256,} \\
& 3.3141,4.7776,3.9725,-0.1110,-1.3841,-0.8745,0.0382,-2.1826,-1.0944,1.4534]^{\mathrm{T}} .
\end{aligned}
$$

Proceeding to an analysis of variance, the factors $A, B$, the interaction $A B$, the quadratic terms $A^{2}$ and $B^{2}$ result important at significance level $\alpha=1 \%$. The estimated coefficients are

$\hat{\beta}=[-0.244506,2.84293,3.35841,0.440448,3.45366,3.66831,0.41685,3.2417,0.0107227,-0.10579]^{\mathrm{T}}$.

So, the final estimated model is

$$
\hat{y}=2.84293 x_{1}+3.35841 x_{2}+3.45366 x_{1}^{2}+3.66831 x_{1} x_{2}+3.2417 x_{2}^{2},
$$

and we see that it provides a very good approximation of the original simulated true model.

Also, it occurs that the combination of the factors levels that maximizes the response $y$ is $x_{1}=1, x_{2}=1, x_{3}=0.0232$ and its maximum value is $y_{\max }=16.3406$.

In a similar way, if we consider as the design matrix the Koshal's design with three factors and 20 runs, 


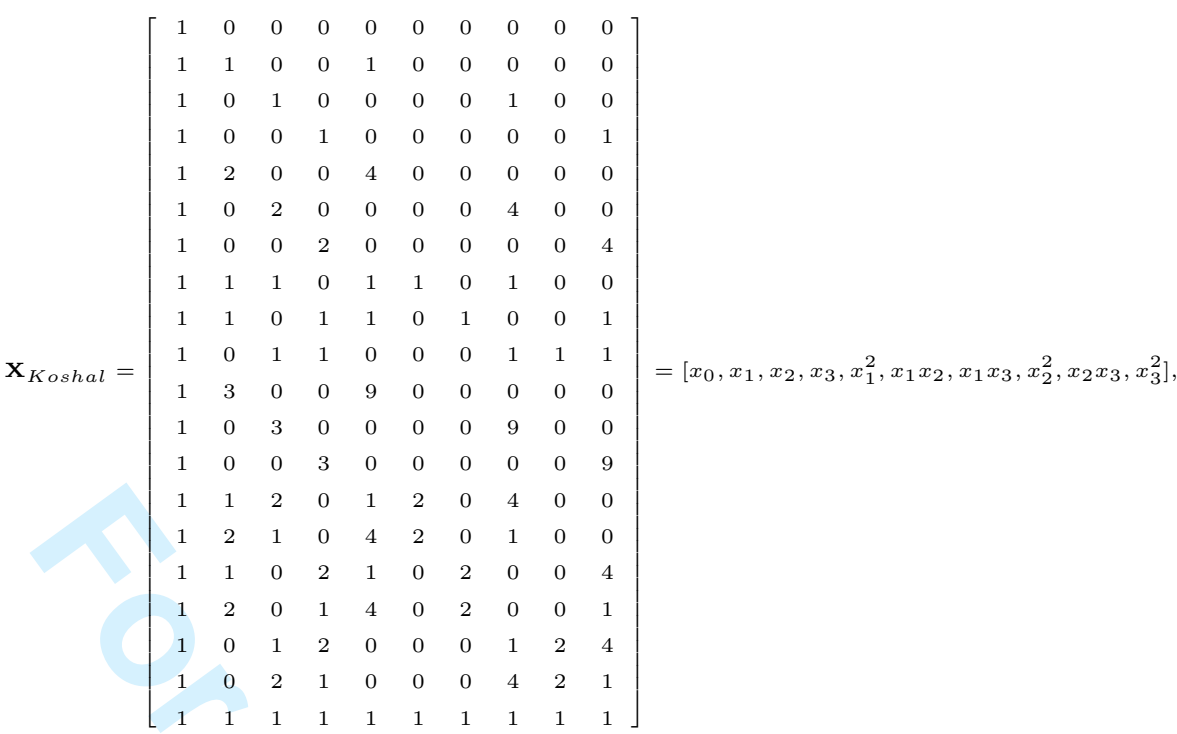

we generate the data according to the simulated model

$$
y=0.5+3 x_{1}+4 x_{2}+3 x_{1}^{2}+3 x_{1} x_{2}+3 x_{2}^{2}+\varepsilon,
$$

with $\varepsilon \sim N_{20}\left(0_{20}, I_{20}\right)$.

A response $y$, obtained by using the above simulated model, is

$$
\begin{aligned}
y= & {[-1.1656,6.6253,7.7877,-0.6465,19.6909,21.6892,0.4624,16.8273,6.6746,7.3133,} \\
& 37.2258,38.9117,2.6832,32.3636,31.6139,7.5668,18.5593,7.4044,19.6677,16.7944]^{\mathrm{T}} .
\end{aligned}
$$

The analysis of variance results that the factors $A, B$, the interactions $A B, B C$, the quadratic terms $A^{2}$ and $B^{2}$ are important at significance level $\alpha=1 \%$. The estimation coefficients are given by the following vector

$$
\hat{\beta}=[-1.18661,5.36746,6.93253,0.0523455,2.47141,1.57721,0.470387,2.15853,-1.02406,0.407094]^{\mathrm{T}} \text {, }
$$


and the final estimated model is

$$
\hat{y}=5.36746 x_{1}+6.93253 x_{2}+2.47141 x_{1}^{2}+1.57721 x_{1} x_{2}+2.15853 x_{2}^{2}-1.02406 x_{2} x_{3} .
$$

In order to maximize the response, the combination of the factors levels should be $x_{1}=$ $3, x_{2}=2.9553, x_{3}=0$, with $y_{\max }=90.4809$.

From the above results, we note that in the first case, we manage to identify exactly the true active effects, while in the second case the interaction $B C$ is overestimated and incorrectly declared active.

\section{ACKNOWLEDGMENTS}

The authors would like to thank the Associate Editor and the anonymous reviewer for their useful comments and suggestions.

\section{BIBLIOGRAPHY}

Box, G. E. P., and Draper, N. R. (1987). Empirical Model Building and Response Surfaces. New York: Wiley.

Box, G. E. P., and Draper, N. R. (2007). Response Surfaces, Mixtures, and Ridge Analyses. New York: Wiley.

Box, G. E. P., and Hunter, J. S. (1957). Multifactor Experimental Designs for Exploring Response Surfaces. The Annals of Mathematical Statistics, 28, 195-241.

Box, M. J., and Draper, N. R. (1971). Factorial Designs, the $\left|\mathbf{X}^{\prime} \mathbf{X}\right|$ Criterion and Some Related Matters. Technometrics, 13, 731-742.

Carla, S. (1997). A Survey of Combinatorial Gray Codes. Society of Industrial and Applied Mathematics Review, 39, 605-629.

Davis, L. (1991). Handbook of Genetic Algorithms. Van Nostrand, Reinhold. 
Drain, D., Carlyle, W. M., Montgomery, D. C., Borror, C., and Anderson-Cook, C. (2004). A Genetic Algorithm Hybrid for Constructing Optimal Response Surface Designs. Quality and Reliability Engineering International, 20, 637-650.

Draper, N. R., and Guttman, I. (1988). An Index of Rotatability. Technometrics, 30, 105-111.

Draper, N. R., and Pukelsheim, F. (1990). Another Look at Rotatability. Technometrics, 32, 195-202.

Edmondson, R. N. (1991). Agricultural Response Surface Experiments Based on Four-Level Factorial Designs. Biometrics, 47, 1435-1448.

Forrest, S. (1993). Genetic Algorithms: Principles of Natural Selection Applied to Computation. Science, 261, 872-878.

Gilmour, S. G. (2004). Irregular Four-Level Response Surface Designs. Journal of Applied Statistics, 31, 1043-1048.

Gilmour, S. G. (2006). Response Surface Designs for Experiments in Bioprocessing. Biometrics, 62, 323-331.

Goldberg, D. E. (1989). Genetic Algorithms in Search, Optimization, and Machine Learning. Addison-Wesley, Reading, MA.

Gupta, T. K., and Dey, A. (1975). On Some New Second Order Rotatable Designs. Annals of the Institute of Statistical Mathematics, 27, 167-175.

Holland, J. H. (1975). Adaptation in Natural and Artificial Systems, an Introductory Analysis with Applications to Biology, Control and Artificial Intelligence. University of Michigan Press, Ann Arbor, Michigan.

Khuri, A. I. (1988). A measure of Rotatability for Response Surface Designs. Technometrics, 30, 95-104. 
Khuri, A. I., and Cornell, J. A. (1996). Response Surfaces. 2nd edition. New York: Dekker. Koshal, R. S. (1933). Application of the Method of Maximum Likelihood to the Improvement of Curves Fitted by the Method of Moments. J. Roy. Statist. Soc. Ser. A, 96, 303-313.

Koukouvinos, C., Mylona, K., and Simos, D. E. (2007). Exploring k-Circulant Supersaturated Designs via Genetic Algorithms. Comput. Statist. Data Anal., 51, 2958-2968.

Koukouvinos, C., Mylona, K., and Simos, D. E. (2008). E( $\left.s^{2}\right)$-Optimal and MinimaxOptimal Cyclic Supersaturated Designs via Multi-Objective Simulated Annealing. J. Statist. Plann. Inference, 138, 1639-1646.

Koukouvinos, C., Mylona, K., and Simos, D. E. (2009). A Hybrid SAGA Algorithm for the Construction of $E\left(s^{2}\right)$-Optimal Cyclic Supersaturated Designs. J. Statist. Plann. Inference, 139, 478-485.

Myers, R.H., and D.C. Montgomery (2002). Response Surface Methodology. 2nd edition. New York: Wiley.

Wang, J. C., and Wu, C. F. J. (1995). A Hidden Projection Property of Plackett-Burman and Related Designs. Statistica Sinica, 5, 235-250. 